\title{
New Subclasses concerning Some Analytic and Univalent Functions
}

\author{
Maslina Darus ${ }^{1}$ and Shigeyoshi Owa ${ }^{2}$ \\ ${ }^{1}$ School of Mathematical Sciences, Faculty of Science and Technology, Universiti Kebangsaan Malaysia, 43600 Bangi, \\ Selangor Darul Ehsan, Malaysia \\ ${ }^{2}$ Department of Mathematics, Faculty of Education, Yamato University, Katayama 2-5-1, Suita, Osaka 564-0082, Japan
}

Correspondence should be addressed to Maslina Darus; maslina@ukm.edu.my

Received 4 April 2017; Accepted 17 July 2017; Published 20 August 2017

Academic Editor: Tomasa Calvo

Copyright (C) 2017 Maslina Darus and Shigeyoshi Owa. This is an open access article distributed under the Creative Commons Attribution License, which permits unrestricted use, distribution, and reproduction in any medium, provided the original work is properly cited.

Considering a function $f(z)=z /\left(1-z^{2}\right)$ which is analytic and starlike in the open unit $\operatorname{disc} U$ and a function $f(z)=z /(1-z)$ which is analytic and convex in $U$, we introduce two new classes $\mathcal{S}_{\alpha}^{*}(\beta)$ and $\mathscr{K}_{\alpha}(\beta)$ concerning $f_{\alpha}(z)=z /\left(1-z^{\alpha}\right)(\alpha>0)$. The object of the present paper is to discuss some interesting properties for functions in the classes $\delta_{\alpha}^{*}(\beta)$ and $\mathscr{K}_{\alpha}(\beta)$.

\section{Introduction and Preliminaries}

Let $\mathscr{A}$ be the class of functions $f(z)$ which are analytic in the open unit disk $U=\{z \in \mathbb{C}:|z|<1\}$ with $f(0)=0$ and $f^{\prime}(0)=1$.

Let $\mathcal{S}$ denote the subclass of $\mathscr{A}$ consisting of functions $f(z) \in \mathscr{A}$ which are univalent in $U$. Also, let $\mathcal{S}^{*}(\beta)$ be the subclass of $\delta$ consisting of $f(z)$ which are starlike of order $\beta(0 \leq \beta<1)$ in $U$. Further, we say that $f(z) \in \mathscr{K}(\beta)$ if $f(z) \in \mathcal{S}$ satisfies $z f^{\prime}(z) \in \mathcal{S}^{*}(\beta)$. A function $f(z) \in \mathscr{K}(\beta)$ is said to be convex of order $\beta$ in $U$ (cf. [1-3]).

With the above definitions for classes $\mathscr{K}(\beta), \mathcal{S}^{*}(\beta), \mathcal{\delta}$, and $\mathscr{A}$, it is known that

$$
\mathscr{K}(\beta) \subset \mathcal{S}^{*}(\beta) \subset \mathcal{S} \subset \mathscr{A}
$$

and $f(z) \in \mathcal{S}^{*}(\beta)$ if and only if $\int_{0}^{z}(f(t) / t) d t \in \mathscr{K}(\beta)$.

The function $f(z)$ given by

$$
f(z)=\frac{z}{1-z^{2}}=z+z^{3}+z^{5}+\cdots \quad(z \in U)
$$

is in the class $\mathcal{S}^{*}(0) \equiv \mathcal{S}^{*}$ and the function $f(z)$ given by

$$
f(z)=\frac{z}{1-z}=z+z^{2}+z^{3}+\cdots \quad(z \in U)
$$

is in the class $\mathscr{K}(0) \equiv \mathscr{K}$.
If we consider the function $f(z)$ given by

$$
f_{\alpha}(z)=\frac{z}{1-z^{\alpha}}=z+\sum_{n=1}^{\infty} z^{1+n \alpha} \quad(z \in U)
$$

for some real $\alpha(0<\alpha \leq 2)$, we discuss some properties between functions $f(z)$ in (2) and (3), where we consider the principal value for $z^{n \alpha}$.

With the function $f(z)$ given by (4), we introduce a class $\mathscr{A}_{\alpha}$ of analytic functions $f(z)$ with series expansion in $U$ such that

$$
f(z)=z+\sum_{n=1}^{\infty} a_{n} z^{1+n \alpha} \quad(z \in U)
$$

for some real $\alpha(0<\alpha \leq 2)$, where we take the principal value for $z^{n \alpha}$. If $f(z) \in \mathscr{A}_{\alpha}$ satisfies

$$
\operatorname{Re}\left(\frac{z f^{\prime}(z)}{f(z)}\right)>\beta \quad(z \in U)
$$

for some real $\beta(0 \leq \beta<1)$, then we say that $f(z) \in \mathcal{S}_{\alpha}^{*}(\beta)$. 
Also, if $f(z) \in \mathscr{A}_{\alpha}$ satisfies

$$
\operatorname{Re}\left(1+\frac{z f^{\prime \prime}(z)}{f^{\prime}(z)}\right)>\beta \quad(z \in U)
$$

for some real $\beta(0 \leq \beta<1)$, then we say that $f(z) \in \mathscr{K}_{\alpha}(\beta)$.

With the above definitions for the classes $\delta_{\alpha}^{*}(\beta)$ and $\mathscr{K}_{\alpha}(\beta)$, we have that $f(z) \in \mathscr{K}_{\alpha}(\beta)$ if and only if $z f^{\prime}(z) \in$ $\mathcal{S}_{\alpha}^{*}(\beta)$ and that $f(z) \in \mathcal{S}_{\alpha}^{*}(\beta)$ if and only if $\int_{0}^{z}(f(t) / t) d t \in$ $\mathscr{K}_{\alpha}(\beta)$.

\section{Some Properties}

In this section, we consider some properties of functions with series expansion given by (4).

Theorem 1. If $f(z)$ is given by (4), then $f(z) \in \mathcal{S}_{\alpha}^{*}((2-\alpha) / 2)$ for $0<\alpha \leq 2$ and $f(z) \in \mathscr{K}_{\alpha}(\alpha)$ for $0<\alpha<1$.

Proof. For $f(z)$ given by (4), we see that $z f^{\prime}(z) / f(z)=1$ for $z=0$ and

$$
\begin{aligned}
\operatorname{Re}\left(\frac{z f^{\prime}(z)}{f(z)}\right) & =\operatorname{Re}\left(\frac{1+(\alpha-1) z^{\alpha}}{1-z^{\alpha}}\right) \\
& =1-\alpha+\alpha \operatorname{Re}\left(\frac{1}{1-z^{\alpha}}\right) \\
& =1-\alpha+\alpha \operatorname{Re}\left(\frac{1}{1-e^{i \alpha \theta}}\right)=\frac{2-\alpha}{2}<1
\end{aligned}
$$

for $z=e^{i \theta}(0<\theta<2 \pi)$. This shows that $f(z) \in \mathcal{S}_{\alpha}^{*}((2-\alpha) / 2)$ for $0<\alpha \leq 2$. Further, we have that $1+z f^{\prime \prime}(z) / f^{\prime}(z)=1$ for $z=0$ and

$$
\begin{aligned}
\operatorname{Re}\left(1+\frac{z f^{\prime \prime}(z)}{f^{\prime}(z)}\right) \\
=\operatorname{Re}\left(\frac{1+(2 \alpha-1) z^{\alpha}}{1-z^{\alpha}}+\frac{\alpha(\alpha-1) z^{\alpha}}{1+(\alpha-1) z^{\alpha}}\right) \\
=3 \alpha-1+2(1-\alpha) \operatorname{Re}\left(\frac{1}{1-z^{\alpha}}\right) \\
\quad-\alpha \operatorname{Re}\left(\frac{1}{1+(\alpha-1) z^{\alpha}}\right) \\
=3 \alpha-1+2(1-\alpha) \operatorname{Re}\left(\frac{1}{1-e^{i \alpha \theta}}\right) \\
\quad-\alpha \operatorname{Re}\left(\frac{1}{1+(\alpha-1) e^{i \alpha \theta}}\right) \\
=2 \alpha-\alpha \frac{1+(\alpha-1) \cos (\alpha \theta)}{1+(\alpha-1)^{2}+2(\alpha-1) \cos (\alpha \theta)}
\end{aligned}
$$

for $z=e^{i \theta}(0<\theta<2 \pi)$. Letting

$$
g(t)=\frac{1+(\alpha-1) t}{1+(\alpha-1)^{2}+2(\alpha-1) t} \quad(t=\cos (\alpha \theta)),
$$

we have that

$$
g^{\prime}(t)=\frac{\alpha(\alpha-1)(\alpha-2)}{\left(1+(\alpha-1)^{2}+2(\alpha-1) t\right)^{2}}>0
$$

$$
(0<\alpha<1) .
$$

Thus, we see that

$$
\operatorname{Re}\left(1+\frac{z f^{\prime \prime}(z)}{f^{\prime}(z)}\right)>\alpha \quad(z \in U)
$$

for $0<\alpha<1$. This completes the proof of the theorem.

Corollary 2. A function

$$
f(z)=\frac{z}{1-\sqrt{z}} \quad(z \in U)
$$

belongs to the class $\mathcal{S}_{1 / 2}^{*}(3 / 4)$ and $\mathscr{K}_{1 / 2}(1 / 2)$.

Next, we discuss some properties of functions $f(z)$ for $\mathscr{A}_{\alpha}$.

Theorem 3. If $f(z)$ given by (5) satisfies

$$
\sum_{n=1}^{\infty}(n \alpha+1-\beta)\left|a_{n}\right| \leq 1-\beta
$$

for some $\beta(0 \leq \beta<1)$, then $f(z) \in \mathcal{S}_{\alpha}^{*}(\beta)$.

The equality holds true for $f(z)$ given by

$$
f(z)=z+\sum_{n=1}^{\infty} \frac{(1-\beta) e^{i \pi}}{n(n+1)(n \alpha+1-\beta)} z^{1+n \alpha} .
$$

Proof. Let the function $f(z)$ be given by (5); then, we have that

$$
\begin{aligned}
\left|\frac{z f^{\prime}(z)}{f(z)}-1\right| & =\left|\frac{\sum_{n=1}^{\infty} n \alpha a_{n} z^{n \alpha}}{1+\sum_{n=1}^{\infty} a_{n} z^{n \alpha}}\right| \leq \frac{\sum_{n=1}^{\infty} n \alpha\left|a_{n}\right||z|^{n \alpha}}{1-\sum_{n=1}^{\infty}\left|a_{n}\right||z|^{n \alpha}} \\
& <\frac{\sum_{n=1}^{\infty} n \alpha\left|a_{n}\right|}{1-\sum_{n=1}^{\infty}\left|a_{n}\right|} \leq 1-\beta
\end{aligned}
$$

if $f(z)$ satisfies (14). This shows that $f(z) \in \mathcal{S}_{\alpha}^{*}(\beta)$. Further, if we consider a function $f(z)$ given by (15), then we see that

$$
\begin{aligned}
\sum_{n=1}^{\infty}(n \alpha+1-\beta)\left|a_{n}\right| & =\sum_{n=1}^{\infty} \frac{1-\beta}{n(n+1)} \\
& =(1-\beta) \sum_{n=1}^{\infty}\left(\frac{1}{n}-\frac{1}{n+1}\right) \\
& =1-\beta .
\end{aligned}
$$

Theorem 4. If $f(z)$ given by (5) satisfies

$$
\sum_{n=1}^{\infty}(n \alpha+1)(n \alpha+1-\beta)\left|a_{n}\right| \leq 1-\beta
$$

for some $\beta(0 \leq \beta<1)$, then $f(z) \in \mathscr{K}_{\alpha}(\beta)$. 
The equality in (18) holds true for $f(z)$ given by

$$
f(z)=z+\sum_{n=1}^{\infty} \frac{(1-\beta)(n \alpha+1) e^{i \pi}}{n(n+1)(n \alpha+1-\beta)} z^{1+n \alpha} .
$$

Further, we obtain the following.

Theorem 5. Let $f(z)$ be given by (5) with $\arg a_{n}=\pi-$ $n \alpha \theta(0<\theta<2 \pi)$. Then, $f(z) \in \mathcal{S}_{\alpha}^{*}(\beta)$ if and only if

$$
\sum_{n=1}^{\infty}(n \alpha+1-\beta)\left|a_{n}\right| \leq 1-\beta
$$

for some $\beta(0 \leq \beta<1)$. The equality holds true for

$$
f(z)=z+\sum_{n=1}^{\infty} \frac{(1-\beta) e^{i(\pi-n \alpha \theta)}}{n(n+1)(n \alpha+1-\beta)} z^{1+n \alpha} .
$$

Proof. Theorem 3 implies that if $f(z)$ satisfies (20), then $f(z) \in \mathcal{S}_{\alpha}^{*}(\beta)$. Next, we suppose that $f(z) \in \mathcal{S}_{\alpha}^{*}(\beta)$. Then,

$$
\operatorname{Re}\left(\frac{z f^{\prime}(z)}{f(z)}\right)=\operatorname{Re}\left(\frac{1+\sum_{n=1}^{\infty}(n \alpha+1) a_{n} z^{n \alpha}}{1+\sum_{n=1}^{\infty} a_{n} z^{n \alpha}}\right) .
$$

If we consider $z=r e^{i \theta}$, then we have that

$$
a_{n} z^{n \alpha}=\left|a_{n}\right| r^{n \alpha} e^{i \pi}=-\left|a_{n}\right| r^{n \alpha} .
$$

Then, we obtain that

$$
\begin{aligned}
\operatorname{Re}\left(\frac{z f^{\prime}(z)}{f(z)}\right) & =\frac{1-\sum_{n=1}^{\infty}(n \alpha+1)\left|a_{n}\right| r^{n \alpha}}{1-\sum_{n=1}^{\infty}\left|a_{n}\right| r^{n \alpha}} \\
& =1-\frac{\sum_{n=1}^{\infty} n \alpha\left|a_{n}\right| r^{n \alpha}}{1-\sum_{n=1}^{\infty}\left|a_{n}\right| r^{n \alpha}}>\beta .
\end{aligned}
$$

This gives us

$$
\frac{\sum_{n=1}^{\infty} n \alpha\left|a_{n}\right|}{1-\sum_{n=1}^{\infty}\left|a_{n}\right|} \leq 1-\beta
$$

that is,

$$
\sum_{n=1}^{\infty}(n \alpha+1-\beta)\left|a_{n}\right| \leq 1-\beta
$$

Thus, $f(z) \in \mathcal{S}_{\alpha}^{*}(\beta)$ if and only if the coefficient inequality (20) holds true.

Further, for the class $\mathscr{K}_{\alpha}(\beta)$, we have the following.

Theorem 6. Let $f(z)$ be given by (5) with $\arg a_{n}=\pi-$ $n \alpha \theta(0<\theta<2 \pi)$. Then, $f(z) \in \mathscr{K}_{\alpha}(\beta)$ if and only if

$$
\sum_{n=1}^{\infty}(n \alpha+1)(n \alpha+1-\beta)\left|a_{n}\right| \leq 1-\beta
$$

for some $\beta(0 \leq \beta<1)$. The equality holds true for

$$
f(z)=z+\sum_{n=1}^{\infty} \frac{(n \alpha+1)(1-\beta) e^{i(\pi-n \alpha \theta)}}{n(n+1)(n \alpha+1-\beta)} z^{1+n \alpha} .
$$

\section{Radius Problems}

In this section, we consider

$$
g(z)=\frac{z}{1-z^{\alpha}} \quad(z \in U)
$$

for some real $\alpha>2$. Then, we say that $g(z) \notin \mathcal{S}_{\alpha}^{*}(\beta)$ and $g(z) \notin \mathscr{K}_{\alpha}(\beta)$ for any real $\beta(0 \leq \beta<1)$.

Now, we derive the following.

Theorem 7. If $g(z)$ is given by (29) with $\alpha>2$, then

$$
\operatorname{Re}\left(\frac{z g^{\prime}(z)}{g(z)}\right)>\frac{1-(\alpha-1) r^{\alpha}}{1+r^{\alpha}} \quad(0<|z|=r<1) .
$$

Proof. For $g(z)$ given by (29), we have that

$$
\frac{z g^{\prime}(z)}{g(z)}=\frac{1+(\alpha-1) r^{\alpha} e^{i \alpha \theta}}{1-r^{\alpha} e^{i \alpha \theta}}=\frac{e^{-i \alpha \theta}+(\alpha-1) r^{\alpha}}{e^{-i \alpha \theta}-r^{\alpha}}
$$

for $z=r e^{i \theta} \in U$. This gives us

$$
\operatorname{Re}\left(\frac{z g^{\prime}(z)}{g(z)}\right)=\frac{1+(\alpha-2) r^{\alpha} \cos \alpha \theta-(\alpha-1) r^{2 \alpha}}{1+r^{2 \alpha}-2 r^{\alpha} \cos \alpha \theta} .
$$

Letting

$$
h(t)=\frac{1+(\alpha-2) r^{\alpha} t-(\alpha-1) r^{2 \alpha}}{1+r^{2 \alpha}-2 r^{\alpha} t} \quad(t=\cos \alpha \theta),
$$

we see that $h^{\prime}(t)>0$. This gives us

$$
\operatorname{Re}\left(\frac{z g^{\prime}(z)}{g(z)}\right)>\frac{1-(\alpha-1) r^{\alpha}}{1+r^{\alpha}}
$$

Corollary 8. If $g(z)$ is given by (29) with $\alpha>2$, then

$$
\operatorname{Re}\left(\frac{z g^{\prime}(z)}{g(z)}\right)>\beta \quad(0 \leq \beta<1)
$$

for $0<|z| \leq \sqrt[\alpha]{(1-\beta) /(\beta+\alpha-1)}<1$.

Proof. If we consider

$$
\operatorname{Re}\left(\frac{z g^{\prime}(z)}{g(z)}\right)>\frac{1-(\alpha-1) r^{\alpha}}{1+r^{\alpha}} \geq \beta,
$$

then

$$
0<r \leq \sqrt[\alpha]{\frac{1-\beta}{\beta+\alpha-1}}<1
$$

Remark 9. If $\beta=0$ in (35), then

$$
0<|z| \leq \sqrt[\alpha]{\frac{1}{\alpha-1}}<1,
$$

and if $\beta=1 / 2$, then

$$
0<|z| \leq \sqrt[\alpha]{\frac{1}{2 \alpha-1}}<1 .
$$




\section{Partial Sums}

Finally, we consider the partial sums of $f(z)$ given by (5). In view of (5), we write

$$
f_{n}(z)=z+a_{n} z^{1+n \alpha} \quad(n=1,2,3, \ldots)
$$

for some real $\alpha(0<\alpha \leq 2)$. Recently, Darus and Ibrahim [4] and Hayami et al. [5] have shown some interesting results for some partial sums of analytic functions.

Now, we derive the following.

Theorem 10. Let $f_{n}(z)$ be given by (40) with $\left|a_{n}\right| \leq 1$. Then,

$$
\begin{aligned}
& \operatorname{Re}\left(\frac{z f_{n}^{\prime}(z)}{f_{n}(z)}\right)>\frac{1-(n \alpha+1)\left|a_{n}\right|}{1-\left|a_{n}\right|} \quad(z \in U), \\
& \operatorname{Re}\left(\frac{z f_{n}^{\prime}(z)}{f_{n}(z)}\right) \geq \frac{1-(n \alpha+1) r^{n \alpha}}{1-r^{n \alpha}} \quad(|z|=r<1) .
\end{aligned}
$$

Proof. It follows that

$$
\begin{aligned}
& \operatorname{Re}\left(\frac{z f_{n}^{\prime}(z)}{f_{n}(z)}\right)=\operatorname{Re}\left(1+\frac{n \alpha a_{n} z^{n \alpha}}{1+a_{n} z^{n \alpha}}\right)=1 \\
& +\operatorname{Re}\left(\frac{n \alpha\left|a_{n}\right| r^{n \alpha}(\cos (n \alpha \theta+\varphi)+i \sin (n \alpha \theta+\varphi))}{1+\left|a_{n}\right| r^{n \alpha} \cos (n \alpha \theta+\varphi)+i\left|a_{n}\right| r^{n \alpha} \sin (n \alpha \theta+\varphi)}\right),
\end{aligned}
$$

where $a_{n}=\left|a_{n}\right| e^{i \varphi}$ and $z=r e^{i \theta}$. This gives us

$$
\begin{aligned}
& \operatorname{Re}\left(\frac{z f_{n}^{\prime}(z)}{f_{n}(z)}\right) \\
& \quad=1+\frac{n \alpha\left|a_{n}\right| r^{n \alpha}\left(\left|a_{n}\right| r^{n \alpha}+\cos (n \alpha \theta+\varphi)\right)}{1+2\left|a_{n}\right| r^{n \alpha} \cos (n \alpha \theta+\varphi)+\left|a_{n}\right|^{2} r^{n \alpha}} .
\end{aligned}
$$

Defining $h(t)$ by

$$
h(t)=\frac{\left|a_{n}\right| r^{n \alpha}+t}{1+2\left|a_{n}\right| r^{n \alpha} t+\left|a_{n}\right|^{2} r^{n \alpha}} \begin{aligned}
& (t=\cos (n \alpha \theta+\varphi)),
\end{aligned}
$$

we have that $h^{\prime}(t)>0$ with $\left|a_{n}\right| \leq 1$.

Thus, we obtain

$$
\operatorname{Re}\left(\frac{z f_{n}^{\prime}(z)}{f_{n}(z)}\right)>1-\frac{n \alpha\left|a_{n}\right| r^{n \alpha}}{1-\left|a_{n}\right| r^{n \alpha}} \quad(0 \leq r<1) .
$$

Making $r \rightarrow 1$ in (46), we see (41). Also letting $\left|a_{n}\right|=1$ in (46), we see (42).

Corollary 11. Let $f_{n}(z)$ be given by (40) with $\left|a_{n}\right| \leq(1-$ $\beta) /(n \alpha+1-\beta)(0 \leq \beta<1)$. Then, $f_{n}(z) \in \mathcal{S}_{\alpha}^{*}(\beta)$.

Proof. Since $\left|a_{n}\right|<1, f_{n}(z)$ satisfies (41).

Therefore, for $\left|a_{n}\right| \leq(1-\beta) /(n \alpha+1-\beta)$, (41) gives us $f_{n}(z) \in \mathcal{S}_{\alpha}^{*}(\beta)$.

\section{Conflicts of Interest}

The authors declare that there are no conflicts of interest regarding the publication of this paper.

\section{Acknowledgments}

The work here is supported by MOHE Grant FRGS/1/2016/ STG06/UKM/01/1.

\section{References}

[1] P. L. Duren, Univalent Functions, vol. 259, Springer-Verlag, New York, Berlin, Heidelberg, Tokyo, 1983.

[2] A. W. Goodman, Geometric Theory of Functions, vol. I and II, Mariner, Tampa, Fla, USA, 1983.

[3] M. I. S. Robertson, "On the theory of univalent functions," Annals of Mathematics. Second Series, vol. 37, no. 2, pp. 374-408, 1936.

[4] M. Darus and R. W. Ibrahim, "Partial sums of analytic functions of bounded turning with applications," Computational \& Applied Mathematics, vol. 29, no. 1, pp. 81-88, 2010.

[5] T. Hayami, K. Kuroki, E. Y. Duman, and S. Owa, "Partial sums of certain univalent functions," Applied Mathematical Sciences, vol. 6, no. 13-16, pp. 779-805, 2012. 


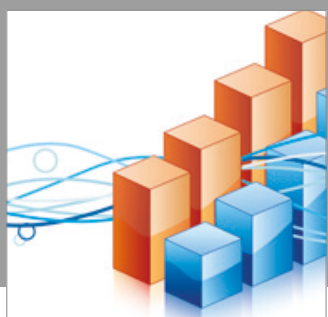

Advances in

Operations Research

vatersals

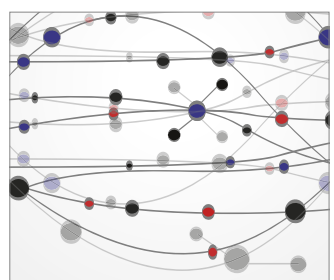

\section{The Scientific} World Journal
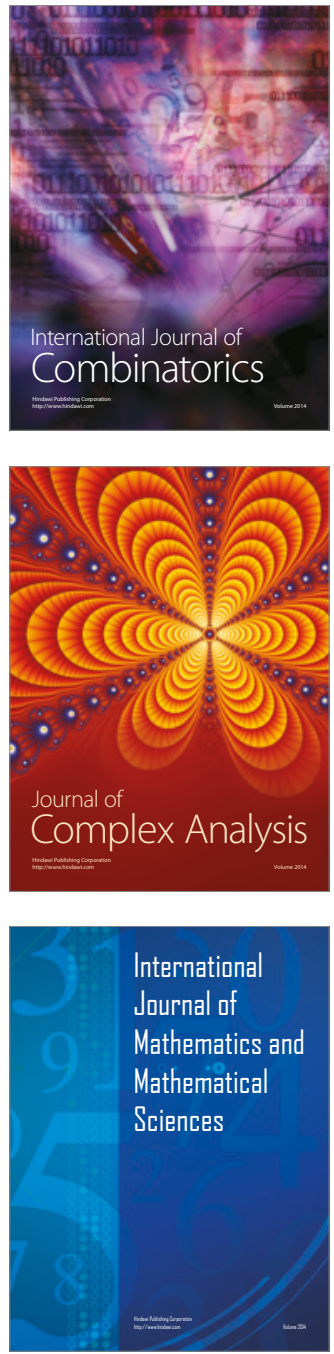
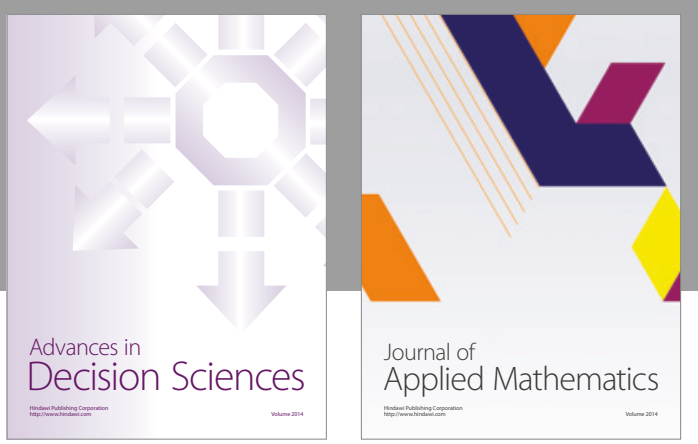

Algebra

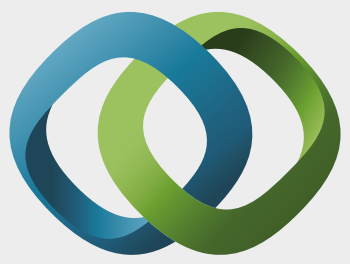

\section{Hindawi}

Submit your manuscripts at

https://www.hindawi.com
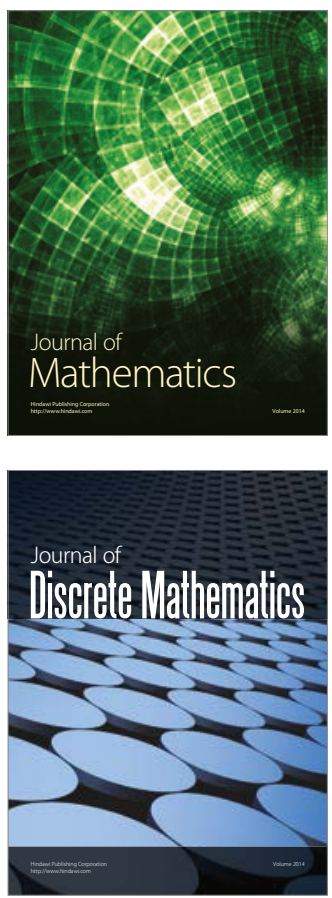

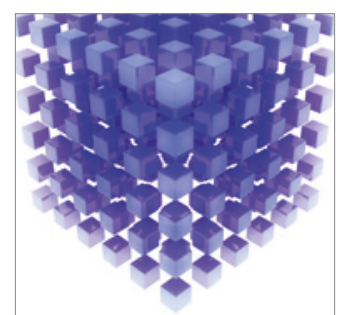

Mathematical Problems in Engineering
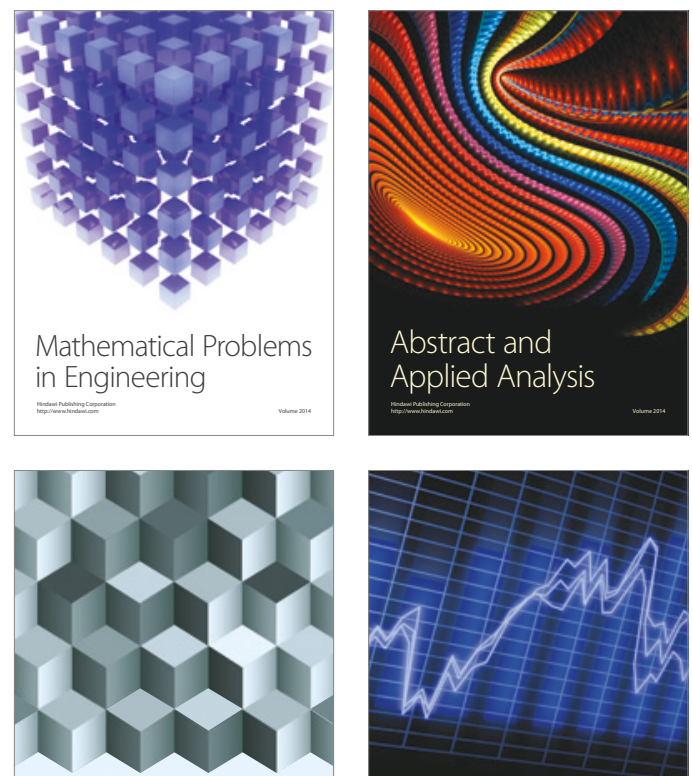

Journal of

Function Spaces

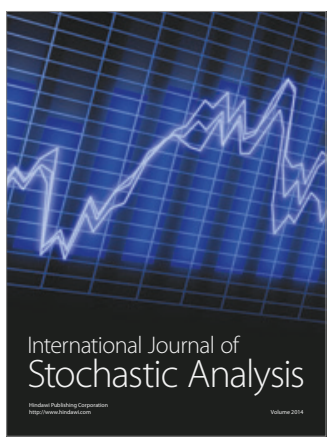

Probability and Statistics
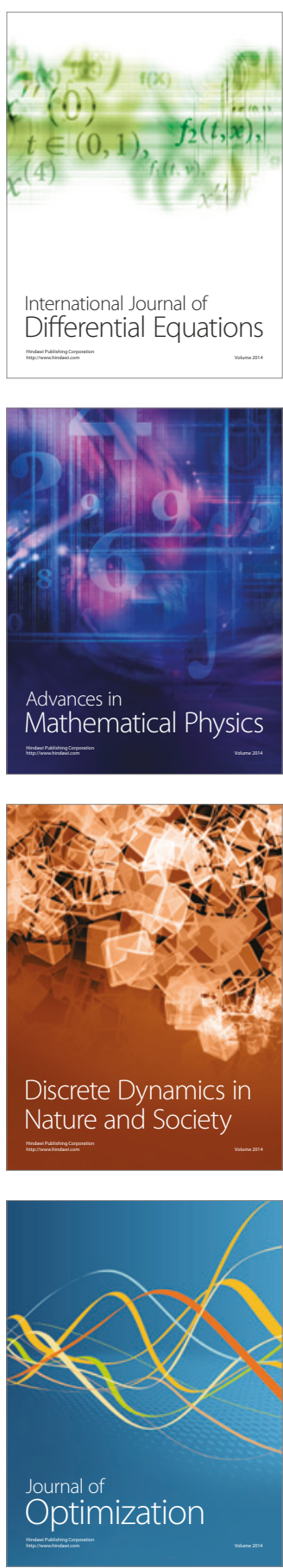